\title{
There Is Something More Important Than Proximal Reflux Episode in the Development of Post-reflux Swallow-induced Peristaltic Wave
}

TO THE EDITOR: I read with interest the paper, "Factors of reflux episodes with post-reflux swallow-induced peristaltic wave in gastroesophageal reflux disease" by Xu et al, ${ }^{1}$ which appeared in Journal of Neurogastroenterology and Motility. The authors should be congratulated for an extensive and careful study. They investigated 269 reflux episodes which consisted of 90 with a postreflux swallow induced peristaltic wave (PSPW) and 179 without a PSPW. In their study, a higher PSPW index was reported to be associated with a higher proximal reflux extent. Generally, if the severity of gastroesophageal reflux disease (GERD) is correlated to a lower PSPW index, then more frequent and higher proximal reflux extent would be seen more frequently in severe GERD. Thus, I wonder why reflux episodes with higher proximal extent have more frequent PSPW episodes.

There is my personal question regarding the authors' views, but I also would like to comment on my views on the factors involving PSPW index in patients with GERD. Twenty-four hour esophageal multichannel intraluminal impedance monitoring combined with $\mathrm{pH}$-metry (MII-pH) study indicates that clearance of refluxed material is handled by a 2-step process (Figure). ${ }^{2} \mathrm{~A}$ rapid volume clearance involving primary and secondary peristalsis occurs first, and slow chemical clearance neutralizing acid follows later. Therefore, esophageal hypomotility disorders are directly linked to impaired volume clearance. Chemical clearance is determined by swallow-induced peristaltic waves evoked by an esophago-salivary reflex mediated through vagal afferents. ${ }^{3}$ A PSPW was defined as an antegrade $50 \%$ drop in impedance occurring within 30 seconds after a reflux event, originating in the most proximal impedance channel, reaching all distal impedance channels, and followed by at least $50 \%$ return to the baseline. ${ }^{4}$ The PSPW index was obtained when dividing the number of PSPWs by the number of reflux events. ${ }^{4}$ The PSPW index evaluating the efficacy of chemical clearance, therefore, has recently been proposed to improve diagnostic yield in patients with GERD symptoms. ${ }^{4}$

In my experience, very low PSPW indices are commonly seen in patients with absent contractility or severe ineffective esophageal motility. Simrén et $\mathrm{al}^{5}$ reported that volume clearance depends on the degree of esophageal hypomotility irrespective of position, but severe hypomotility disorder has a greater impact on chemical clearance only in the supine position. A recent study using both high-resolution manometry and MII-pH reported that ineffective esophageal motility is related to chemical clearance dysfunction as measured lower PSPW index. ${ }^{6}$ In the study, GERD patients with IEM had a lower PSPW index (11.1\%) compared to those with normal motility (17.1\%). I have also often experienced that GERD patients' PSPW indices are low when analyzing MII-pH data for patients with complicated diabetes or Sjogren's syndrome. Further MII-pH study should confirm whether impaired chemical clearance exists in patients with these conditions. However, it is intuitive that these conditions are strongly linked with vagal nerve dysfunction or impaired salivary secretion, suggestive of impaired esophago-salivary reflex.

Tae Hee Lee Institute for Digestive Research, Digestive Disease Center Soonchunhyang University Seoul Hospital, Seoul, Korea

1. Xu H, Ye B, Ding Y, Wang M, Lin L, Jiang L. Factors of reflux episodes with post-reflux swallow-induced peristaltic wave in gastroesopha- 


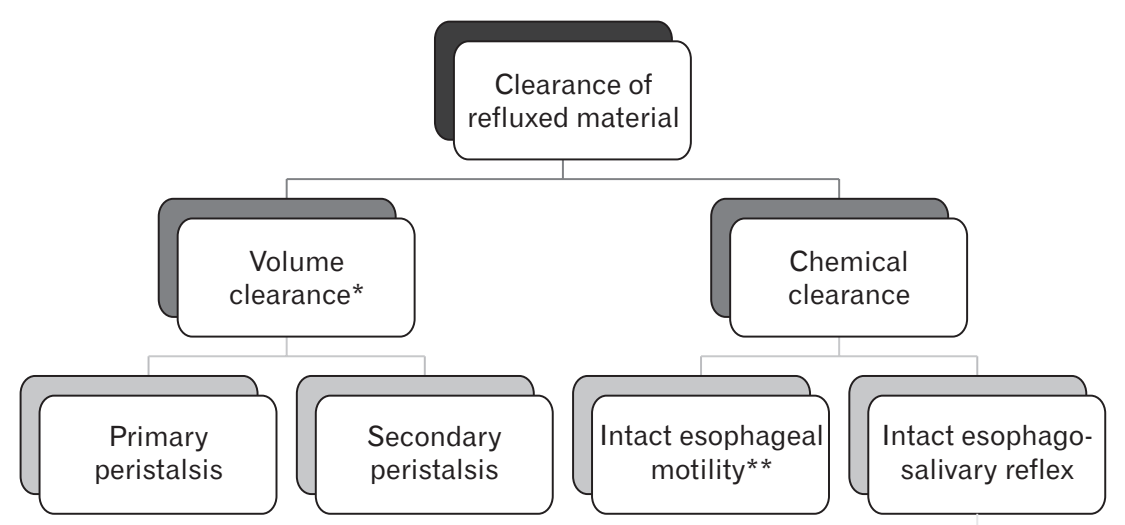

*Esophageal hypomotility is related to impaired volume clearance irrespective of position.

**Esophageal hypomotility impairs chemical clearance only in the supine position.

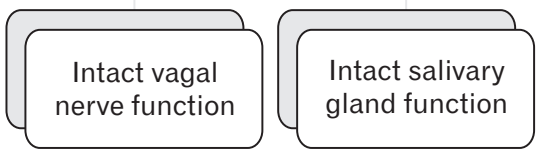

Figure. A 2-step clearance of refluxed material. geal reflux disease. J Neurogastroenterol Motil 2020;26:378-383.

2. Frazzoni M, Manta R, Mirante VG, Conigliaro R, Frazzoni L, Melotti G. Esophageal chemical clearance is impaired in gastro-esophageal reflux disease--a 24-h impedance-pH monitoring assessment. Neurogastroenterol Motil 2013;25:399-406, e295.

3. Shafik A, El-Sibai O, Shafik AA, Mostafa R. Effect of topical esophageal acidification on salivary secretion: identification of the mechanism of action. J Gastroenterol Hepatol 2005;20:1935-1939.

4. Frazzoni M, de Bortoli N, Frazzoni L, Tolone S, Savarino V, Savarino E. Impedance-pH monitoring for diagnosis of reflux disease: new perspectives. Dig Dis Sci 2017;62:1881-1889.
5. Simrén M, Silny J, Holloway R, Tack J, Janssens J, Sifrim D. Relevance of ineffective oesophageal motility during oesophageal acid clearance. Gut 2003;52:784-790.

6. Dao HV, Matsumura T, Kaneko T, et al. Impact of ineffective esophageal motility on chemical clearance in patients with gastroesophageal reflux symptoms. Dis Esophagus 2020;33:doaa026.

Financial support: This work was supported by the Soonchunhyang University Research Fund.

Conflicts of interest: None. 